\title{
Preterm Infant Neurodevelopmental Care Training Program and Mother-Infant Attachment
}

\author{
Fatemeh Fallah Rostami ${ }^{1}$, Farin Soleimani ${ }^{*}{ }^{\mathbb{D}}$, Mehdi Norouzi $^{3}$, Nikta Hatamizadeh ${ }^{2}$, Jamileh \\ Mokhtarinouri $^{4}$, Marjan Poshtmashhadi ${ }^{2}$
}

\begin{abstract}
Objectives: Preterm birth and admission to the neonatal intensive care unit (NICU) would disrupt mother-infant attachment. Neurodevelopmental care training and support of family programs are essential for the family of such infants. The purpose of this study was to investigate the effect of neurodevelopmental care training program for mothers with preterm infants on mother-infant attachment at one month's age.

Materials and Methods: Study population included all the mothers of preterm infants born in the hospitals of Tehran in 2018. The research was designed as a multicenter cluster clinical trial and four hospitals were randomly selected and divided into intervention and control groups. Before the intervention, the mother-infant attachment was measured by the Maternal-Postnatal Attachment Scale (MPAS). The mothers in the intervention group received a 12 -session preterm infant neurodevelopmental care training program while the control group only received the routine care in the unit. Finally, the mother-infant attachment was re-measured in both groups at the one month corrected age.

Results: No significant difference was observed in the attachment score before and after the intervention in the control group but the mother-infant attachment score was significantly different in the intervention group ( $57.75 \pm 11.09 \mathrm{vs}$. $78.27 \pm 4.54)$.

Conclusions: The neurodevelopmental care training program was effective and increased the mother-infant attachment rate.

Keywords: Preterm infant, Neurodevelopmental care, Maternal-postnatal attachment scale, Attachment
\end{abstract}

\section{Introduction}

Preterm birth has been increasing over the last two decades and has led to greater admissions to the neonatal intensive care units (NICUs). There are approximately 13 million preterm births across the world with 5\%-12\% and $40 \%$ prevalence in developed and developing countries, respectively (1).

The prevalence of preterm birth is $7 \%-8 \%$ in Iran. On average, about $9 \%$ of the infants need to be admitted to the NICU (2). Admission to the NICU causes early mother-infant separation. This separation period begins immediately after childbirth. The mother is usually discharged while her premature infant will be kept in the unit for 1 to 3 months. It not only affects the preterm infant who needs maternal interaction but has also a deep impact on the mother and may expose her to the risk of depression and stress (3). In addition, the majority of mothers do not receive any training in relation to complications from preterm birth.

The environment of the NICUs can increase the mothers' stress that they experience in addition to preterm birth and low birth weight. Early separation from the infant and stressors, separately or together, can interfere with mother-infant attachment and cause numerous problems for the mother and development of the preterm infant in the future.

The theory of attachment is the joint work of Mary Ainsworth (1982) and John Bowlby (1969) who defined attachment as the patterns of lifelong connections. In Bowlby's view, attachment is one of the fundamental needs of the human being. By definition, attachment refers to the emotional bond between a growing infant and their caregiver which is of vital importance for healthy development. He also proposed a psychoanalytic view stating that early childhood experiences would have a great impact on a child's behavioral development. Sensitive maternal behavior would reinforce safe attachment relationships and mutually persuasive interactions would shape the attachment with a more dependable and reliable interactional model $(4,5)$.

The development of the infant relies on the quality of continuous interaction with the environment which includes family, social interaction, and attachment to preliminary care. Maternal ability to understand and 
respond to the infant's behavioral signals is important for creating safe attachment.

In the first year of life, infants communicate through eyes and movements which help them to understand the social relationships that are vital for development.

The fact that the infant seeks their caregiver, usually the mother, and cling to her does corroborate an attachment between them. Infants typically begin to show this behavior by the time they are one month old, which is designed to accelerate closeness to the desired person (6).

Some researchers found that if the mother has skinto-skin and body contact with her infant, she will form a stronger bond and may perform her care duties more carefully. Various studies confirmed that preterm infants might have lower consciousness and responsiveness and less engagement in social games compared to normal infants (7).

Research has shown that creating safe attachment during infancy will allow the infants to establish close relationships with their parents in higher ages and, on the contrary, children with less attachment and loving relationships will have lower emotional and mental development and show more student-defector behavior, poor social interactions, aggressive behavior, and decreased ability to establish long-term relationships (8).

The development of the right hemisphere depends on the safe attachment between the infant and the early caregiver. The early relationship can have either a positive or negative impact on the brain's structure and thus on the emotional-behavioral development of the infants. If the attachment is not safe, the limbic system will not be activated, leading to anxiety in the future. In addition, infants with unsafe attachments are more likely to suffer behavioral disorders, lower intelligence, and social incompatibility and are more exposed to mental problems including depression, anxiety, and negative emotional reactions $(9,10)$.

Although many studies addressed the positive impact of establishing parent-preterm infant attachment, most of them suffer the absence of a structured supportive plan.

Neurodevelopmental care is a type of supportive care that reduces infants' stress and pain while increasing the parental presence at their bedside, strengthening motherinfant attachment and bond, and influencing various aspects of development.

Family-centered neurodevelopmental care has recently become common as a new paradigm in the NICUs which considers family as the center of neonatal care and support. Further, enablement and empowerment are two basic concepts of family-centered care. For enablement, by training and providing parents with an opportunity, professionals make them acquire the necessary abilities and competences to meet their infant's needs. Empowerment is interpreted as the parents' sense or acquisition of control over the situation and the ability to overcome the tension from infant admission to the NICUs $(11,12)$.
It should be noted that in the Iranian culture, mothers are more inclined to support and take care of their infants and the caregiver-infant ratio is low in the NICUs. Thus, mothers can be trained through neonatal care programs. Considering the above-mentioned explanations, the present study employed such interventions for mothers with preterm infants and investigated the effects of these structured educational interventions on motherinfant attachment. The interventions included the components of neurodevelopmental care (i.e., caring for the environment, noise, touch, smell, light, taste, sleep, movement, and position, deep sleep, stress and pain reduction, skincare, nutrition and breastfeeding, and temperature). Furthermore, various information was provided, including familiarity with the NICU environment and preterm infant, the identification of the symptoms of infant stress and the consequences of prematurity, skill training in emotional support for infants, and the acquisition of the breastfeeding-kangaroo mother care skill.

\section{Materials and Methods}

The study design was a multicenter cluster clinical trial with a control group. Four hospitals with matched neurodevelopmental care (13-15) were selected using a random cluster sampling method. Then, 72 dead mother-infant cases who met the inclusion criteria were selected by means of a purposive sampling technique. The inclusion criteria were being within the range of 28 34 week of the gestational age, having a birth weight of $<2500$ g, having reading and writing literacy and ability to speak Persian, and being a resident of Tehran Province. On the other hand, the exclusion criteria included the absence of intraventricular hemorrhage grade III and IV, hydrocephaly, the retinopathy of prematurity grade 3 or higher, and NICU hospitalization $<1$ week (to have enough time to train the mothers).

After obtaining the consent form, the Persian version of the Maternal-Postnatal Attachment Scale (MPAS) was administered to both intervention and control groups. Then, mothers received the neurodevelopmental care training program during their infant's hospitalization. The main components of the training and their subcomponents are shown in Table 1. After the intervention, the MPAS was administered again to both groups at the corrected gestational age of one month.

The MPAS is designed for those within the age range of 0 to 36 months and is administered to the mother or any individual who spends the most time with the infant. It has 19 items and 3 subscales including the quality of attachment (items 3, 4, 5, 6, 7, 10,14, 18, and 19), the absence of hostility (items 1, 2, 15, 16, and 17), and pleasure in interaction (items $8,9,11,12$, and 13).

It is scored based on the Likert-type scale and a high score indicates high mother-infant attachment. Moreover, the total score of the 3 subscales shows the total attachment 
Table 1. The Items of the Neurodevelopmental Care Program for Mothers With Preterm Infants in the NICU

\begin{tabular}{ll}
\hline Row & Content \\
\hline 1 & Educational interventions for environmental noise reduction \\
2 & Educational interventions for environmental light reduction \\
3 & Educational interventions for olfactory and gustatory senses \\
4 & Educational interventions to provide safe and restful sleep \\
5 & Educational interventions to move, position, and manipulate the infant \\
6 & Educational supportive interventions to reduce pain and stress \\
7 & Educational interventions for skin care and Kangaroo-Mother Care \\
8 & Educational interventions to optimize nutrition \\
9 & Educational interventions to recognize the stress behavior \\
10 & Educational interventions to have parental communication and interaction with existing conditions and receive support and necessary \\
11 & information \\
12 & Familiarity with preterm infant and the consequences of prematurity \\
\hline
\end{tabular}

Note. NICU: Newborn intensive care unit.

score and the scores range from 19 to 95 (16).

Prior to the intervention, all infants were evaluated using the New Ballard Score which determines the gestational age through the assessment of the neuromuscular and physical maturity of the newborns. This system, which was developed by Ballard in 1979 through the recapitulation of Dubowitz criteria, uses physical and neuromuscular criteria to determine neonatal maturity. Six physical and 6 neuromuscular criteria are measured in this scoring system. Additionally, it is the most valid test for determining the gestational age of preterm infants (17).

The mothers, the outcome assessor, and the data analyzer were blind to the groupings of the subjects. This research employed analysis of covariance (ANCOVA) and assumptions related to the model and normal distribution of the scores were reviewed before testing the hypotheses.

\section{Results}

Tables 2 and 3 present demographic characteristics of the parents and infants. In both groups, neonatal weight was $<2500 \mathrm{~g}$ and the gestational age at the time of birth was 32-33 weeks.

In addition, the normality test for all variables under study was $P=0.82$ and $P=0.77$ in the pretest and posttest and the homogeneity of variances in the MPAS was $P=0.26$. Therefore, the data had a normal distribution and one of the assumptions of ANCOVA statistical models was observed as well $(P>0.05)$.

Based on the results of the independent $t$ test (Table 4), the infants' scores on the New Ballard neuromuscular maturity score were not different across intervention and control groups and both groups were homogeneous in these terms at birth $(P=0.36)$.

The results further (Table 5) indicated that the post-

Table 2. Demographic Characteristics of the Parents

\begin{tabular}{|c|c|c|c|c|c|}
\hline \multirow{3}{*}{ Variable } & & \multicolumn{4}{|c|}{ Group } \\
\hline & & \multicolumn{2}{|c|}{ Intervention } & \multicolumn{2}{|c|}{ Control } \\
\hline & & Mean & SD & Mean & SD \\
\hline \multirow[t]{2}{*}{ Maternal age } & & 28.45 & 4.18 & 28.34 & 4.32 \\
\hline & & Number & $\%$ & Number & $\%$ \\
\hline \multirow{2}{*}{ Mother's education } & High school diploma and lower & 26 & 72.22 & 28 & 77.77 \\
\hline & University degree & 10 & 36 & 8 & 22.22 \\
\hline \multirow{2}{*}{ Father's education } & High school diploma and lower & 26 & 72.22 & 25 & 69.44 \\
\hline & University degree & 10 & 27.77 & 11 & 30.55 \\
\hline \multirow{2}{*}{ Mother's employment } & Housewife & 32 & 88.88 & 33 & 91.66 \\
\hline & Employed & 4 & 11.11 & 3 & 8.33 \\
\hline \multirow{3}{*}{ Father's employment } & Worker & 6 & 16.66 & 7 & 19.44 \\
\hline & Civil servant & 8 & 22.22 & 10 & 27.77 \\
\hline & Self-employed & 22 & 61.11 & 19 & 52.77 \\
\hline \multirow{2}{*}{ Type of delivery } & NVD & 5 & 13.88 & 8 & 22.22 \\
\hline & C-section & 31 & 86.11 & 28 & 77.77 \\
\hline \multirow{2}{*}{ Gender } & Male & 19 & 52.77 & 16 & 44.44 \\
\hline & Female & 17 & 47.22 & 20 & 55.55 \\
\hline
\end{tabular}

Note. SD: standard deviation; NVD: normal vaginal delivery; C-section: cesarean section.

$P$ value $=0.384$ 
Table 3. Demographic Characteristics of the Infants

\begin{tabular}{lcccc}
\hline \multirow{2}{*}{ Variable } & \multicolumn{4}{c}{ Group } \\
\cline { 2 - 5 } & \multicolumn{2}{c}{ Intervention } & \multicolumn{2}{c}{ Control } \\
\cline { 2 - 5 } & Mean & SD & Mean & SD \\
\hline Weight $(\mathrm{g})$ & 1895.02 & 10.09 & 1706.50 & 11.38 \\
\hline Height $(\mathrm{cm})$ & 43.02 & 6.14 & 42.90 & 5.36 \\
\hline Head circumference $(\mathrm{cm})$ & 30.20 & 5.13 & 29.75 & 5.19 \\
\hline Apgar score & & & & \\
\hline $1^{\text {st }}$ minute & 8.50 & 0.93 & 8.30 & 1.07 \\
\hline $5^{\text {th }}$ minute & 9.30 & 1.13 & 9.30 & 0.29 \\
\hline Gestational age & 33.04 & 4.02 & 32.60 & 5.13 \\
\hline
\end{tabular}

Note. SD: Standard deviation.

Table 4. New Ballard Neuromuscular Maturity Scores

\begin{tabular}{lccccc}
\hline Group & Number & Mean & SD & $\begin{array}{c}\text { Skewness } \\
\text { Coefficient }\end{array}$ & $\begin{array}{c}\text { Kurtosis } \\
\text { Coefficient }\end{array}$ \\
\hline Control & 36 & 12.16 & 2.69 & 0.12 & 0.06 \\
Intervention & 36 & 14.20 & 3.82 & 0.61 & 0.56 \\
\hline
\end{tabular}

Note. SD: Standard deviation.

intervention mean score of mother-infant attachment was 62.69 and 78.27 in control and intervention groups, respectively. Moreover, the scores of skewness and kurtosis coefficients suggested that there was no outlier score in the data, indicating that the parametric statistical assumption was observed as well.

According to Table 6, the single-variable covariance analysis of the effects of the neurodevelopmental care for preterm infants on mother-infant attachment demonstrated that $43 \%$ of the attachment difference between the two groups was associated with the interventional program $(\mathrm{f}=52.02, P<0.001)$.

\section{Discussion}

The findings of this study showed that the neurodevelopmental care training program was effective in mother-infant attachment in the intervention group after moderating the pretest effect.

In a study on the effects of breastfeeding-kangaroo mother care on parental outcomes at the corrected age of 37 weeks and 3 months of age on 146 infants, weighing 530$1720 \mathrm{~g}$, and 25-34 weeks of the gestational age, Feldman et al reported the positive and significant effect of kangaroo mother care on parent-infant interaction. They concluded that the mother's active caring and physical bonding might reduce maternal depression while increasing her knowledge of the infant's interactional signals (18), which is in line with the findings of the present study.

Similarly, Sohrabi et al conducted a clinical trial to examine the effect of infant massage by a mother, as a major component of developmental care, on the attachment behaviors of mothers whose infants were hospitalized in the NICU. They selected and divided 42 mother-infant cases into control and experimental groups. The infants were all term cases with a birth weight of $\geq 2500$ g. Further, the maternal attachment behaviors were observed and recorded using the attachment behavior measurement scale 5 days after the intervention. Maternal attachment mean scores represented a statistically significant difference across the groups (19), which is consistent with our findings.

The studies of Borimnejad et al and Karbandi et al in relation to the effects of empowerment programs on mothers' attachment to their preterm infants in the NICU demonstrated an increase in mother-infant attachment following the implementation of the empowerment program. As part of developmental care, it was a multistage behavioral training program for preterm infants and how to provide care for them. Both studies measured motherinfant attachment before the discharge using Avant's mother-infant attachment behavior questionnaire $(20,21)$.

However, contrary to our study, Meijssen et al did not find a significant difference in maternal attachment between intervention and control groups following an early intervention for preterm infants (22). Their supportive intervention was based on the Newborn Individualized Developmental Care and Intervention Program (NIDCAP) with the purpose of helping parents identify neonatal behaviors and self-regulation. The

Table 5. Maternal-Postnatal Attachment Scale Scores in 2 Groups

\begin{tabular}{lccccc}
\hline Group & Stage & Mean & SD & Skewness Coefficient & Kurtosis Coefficient \\
\hline \multirow{2}{*}{ Control } & At birth & 49.77 & 19.84 & 0.41 & 0.98 \\
\cline { 2 - 6 } & One month corrected age & 62.69 & 7.83 & 0.61 & 0.75 \\
\multirow{2}{*}{ Intervention } & At birth & 57.75 & 11.09 & 0.15 & 0.18 \\
\hline
\end{tabular}

Note. SD: Standard deviation.

Table 6. The Effects of Training on Mother-infant Attachment With the Modification of the Pretest Effect

\begin{tabular}{lccccc}
\hline Source of Changes & Sum of Squares & Degree of Freedom & Mean of Squares & F & Significance Level \\
\hline Group & 2793.36 & 1 & 2793.36 & 52.02 & 0.00 \\
Error & 3704.97 & 69 & 53.69 & - & - \\
Total & 9945.98 & 71 & - & - & - \\
\hline
\end{tabular}


sample infants were under 32 weeks old and weighed less than 1500 g. Furthermore, the maternal attachment was measured at the corrected age of 18 months using the Working Model of the Child Interview questionnaire and the mothers were asked about their feelings toward their children. The difference between the results of this study and our study is attributed to the selection of high-risk infants (less weight and more prematurity), the difficulty of behavior-reading and self-regulatory signals used in the intervention, different tests, and higher age of infants.

Glasser et al also reported that the attachment scores one week after delivery were significantly lower in the cesarean section group compared to the normal vaginal delivery group, but the difference was no longer significant at the age of 6 weeks (23).

In another study, Hasanpour et al found no significant difference in mother-infant attachment scores at the adjusted age of one year. They assessed motherinfant attachment with 1-year-old infants through the implementation of the NIDCAP in Al-Zahra hospital of Tabriz and did not find any significant difference in this regard. It is worth noting that the neurodevelopmental care program was implemented by ward nurses in this study (6).

Kleberg et al investigated the effect of NIDCAP on developmental outcomes, neonatal behavior, and motherinfant interaction at the age of 3 years. To this end, they studied 42 preterm infants, all of whom were normal and weighted $\leq 1500 \mathrm{~g}$ and the intervention group received NIDCAP for 3 days after birth. The results showed that the NIDCAP mothers had more physical and eye contact with their infants who were also extrovert, easily expressed their feelings, and demonstrated less behavioral problems (24).

In general, all of the above-mentioned studies indicated the disruption of mother-infant attachment because of special physical conditions, as well as the separation of preterm infants from the family for a long time. Mothers may also suffer stress because of their infants' conditions and their own inability to take care of their infants and these factors work together to put mother-infant attachment in danger.

\section{Limitations of the Study}

The limitations of this study were the exclusion of fathers and no recording of hours spent by mothers at their infant's bedside during the NICU stay.

\section{Conclusions}

The results of the present study revealed that the neurodevelopmental care training program for mothers with preterm infants hospitalized in the NICU was effective in mother-infant attachment at the age of one month in the intervention group.

The hospitalization of the preterm infant disrupts the establishment of mother-infant attachment and the quality of maternal care for the infant, thus it seems that motherinfant interaction should be increased by implementing and teaching developmental care to the mothers of preterm infants in order to raise the level of mother-infant attachment. In addition, the policymakers of this field are recommended to implement such programs in the NICUs in order to empower parents to effectively communicate with their infants and learn necessary caregiving skills. Accordingly, parents are engaged in this process and will not simply be the observers because parent-infant communication contributes to the improved quality of the parental role and a sense of adequacy.

\section{Conflict of Interests}

Authors declare that they have no conflict of interests.

\section{Ethical Issues}

The clinical trial and the ethics codes of this research are 1N20180125038502IRCT and IR.USWR.REC.1396.252, respectively, which were obtained from the University of Social Welfare and Rehabilitation Sciences.

\section{References}

1. Villar J, Merialdi M, Gulmezoglu AM, et al. Characteristics of randomized controlled trials included in systematic reviews of nutritional interventions reporting maternal morbidity, mortality, preterm delivery, intrauterine growth restriction and small for gestational age and birth weight outcomes. J Nutr. 2003;133(5 Suppl 2):1632S-1639S. doi:10.1093/jn/133.5.1632S

2. Afrakhteh M, Valayi N. Preterm Labor Incidence and Related Factores. Pejouhandeh. 2003:341-343. [Persian].

3. Zamanzadeh V, Valizadeh L, Negarandeh R, Monadi M, Azadi A. Factors influencing men entering the nursing profession, and understanding the challenges faced by them: Iranian and developed countries' perspectives. Nurs Midwifery Stud. 2013;2(4):49-56. doi:10.5812/nms.12583

4. Bretherton I. The origins of attachment theory: John Bowlby and Mary Ainsworth. Dev Psychol. 1992;28(5):759775.

5. Bowlby J. A Secure Base: Parent-Child Attachment and Healthy Human Development. New York: Basic Books; 1988.

6. Hasanpour S, Ouladsahebmadarek E, Hosseini MB, Mirghafourvand M, Heidarabadi S, Asghari Jafarabadi M. Mother-infant attachment at the age of 1 year in recipients of developmental care after preterm birth. International Journal of Women's Health and Reproduction Sciences. 2018;6(1):90-96. doi:10.15296/ijwhr.2018.16

7. Brett J, Staniszewska S, Newburn M, Jones N, Taylor L. A systematic mapping review of effective interventions for communicating with, supporting and providing information to parents of preterm infants. BMJ Open. 2011;1(1):e000023. doi:10.1136/bmjopen-2010-000023

8. Bretherton I, Munholland KA. Internal working models in attachment relationships: elaborating a central construct in attachment theory. In: Cassidy J, Shaver PR, eds. Handbook 
of Attachment: Theory, Research, and Clinical Applications. The Guilford Press; 2008:102-127.

9. Malekpour M. Effects of attachment on early and later development. The British Journal of Development Disabilities. 2007;53(105):81-95. doi:10.1179/096979507799103360

10. Pennestri MH, Gaudreau H, Bouvette-Turcot AA, et al. Attachment disorganization among children in neonatal intensive care unit: preliminary results. Early Hum Dev. 2015;91(10):601-606. doi:10.1016/j. earlhumdev.2015.07.005

11. Als H, Lawhon G, Duffy FH, McAnulty GB, GibesGrossman R, Blickman JG. Individualized developmental care for the very low-birth-weight preterm infant. Medical and neurofunctional effects. JAMA. 1994;272(11):853-858.

12. Altimier L, Phillips $\mathrm{R}$. The neonatal integrative developmental care model: advanced clinical applications of the seven core measures for neuroprotective familycentered developmental care. Newborn Infant Nurs Rev. 2016;16(4):230-244. doi:10.1053/j.nainr.2016.09.030

13. Eskandari Z, Razavi Nejad M, Akrami F, Almasi-Hashiani A, Heidarzadeh M. Assessing staff-oriented care with developmental support approach in Iranian NICUs. J Matern Fetal Neonatal Med. 2019;32(6):1009-1013. doi:10 $.1080 / 14767058.2017 .1397127$

14. Razavi Nejad M, Eskandari Z, Heidarzadeh M, Afjeh A, Almasi-Hashiani A, Akrami F. Assessing infant-oriented care with developmental support approach in Iranian NICUs. J Matern Fetal Neonatal Med. 2018;31(14):18511855. doi:10.1080/14767058.2017.1330879

15. Razavi Nejad M, Heidarzadeh M, Mohagheghi P, Akrami F, Almasi-Hashiani A, Eskandary Z. Assessment of Physical Environment of Iran's Neonatal Tertiary Care Centers from the Perspective of the Neonatal Individualized Developmental Care. Iranian Journal of Neonatology IJN. 2017;8(4):20-25. doi:10.22038/ijn.2017.21258.1240

16. Ghadery-Sefat A, Abdeyazdan Z, Badiee Z, ZarghamBoroujeni A. Relationship between parent-infant attachment and parental satisfaction with supportive nursing care. Iran J Nurs Midwifery Res. 2016;21(1):71-76. doi:10.4103/1735-9066.174756

17. Ballard JL, Khoury JC, Wedig K, Wang L, Eilers-Walsman BL, Lipp R. New Ballard Score, expanded to include extremely premature infants. J Pediatr. 1991;119(3):417423. doi:10.1016/s0022-3476(05)82056-6

18. Feldman R, Eidelman AI, Sirota L, Weller A. Comparison of skin-to-skin (kangaroo) and traditional care: parenting outcomes and preterm infant development. Pediatrics. 2002;110(1 Pt 1):16-26. doi:10.1542/peds.110.1.16

19. Sohrabi S, Ahmadi Z, Mosayebi Z, Haghani H. Effect of infant massage by mothers on maternal attachment behavior in infants hospitalized in neonatal care units. Hayat. 2014;20(2):59-68. [Persian].

20. Borimnejad L, Mehrnoosh N, Fatemi NS, Haghani H. Impacts of creating opportunities for parent empowerment on maternal stress: a quasi-experimental study. Iran J Nurs Midwifery Res. 2013;18(3):218-221.

21. Karbandi S, Momenizadeh A, Hydarzadeh M, Mazlom S, Hasanzadeh M. The Effect of Mother Empowerment Program on Mothers' Attachment to their Hospitalized Premature Neonates. Evidence Based Care. 2015;5(2):7-14. doi:10.22038/ebcj.2015.4498

22. Meijssen D, Wolf MJ, van Bakel H, Koldewijn K, Kok J, van Baar A. Maternal attachment representations after very preterm birth and the effect of early intervention. Infant Behav Dev. 2011;34(1):72-80. doi:10.1016/j. infbeh.2010.09.009

23. Glasser R. Method of Delivery and Maternal Infant Attachment. ProQuest; 2007.

24. Kleberg A, Westrup B, Stjernqvist K. Developmental outcome, child behaviour and mother-child interaction at 3 years of age following Newborn Individualized Developmental Care and Intervention Program (NIDCAP) intervention. Early Hum Dev. 2000;60(2):123-135. doi:10.1016/s0378-3782(00)00114-6 